Lindquist Anthea (Orcid ID: 0000-0002-8662-5188)

Poulton Alice (Orcid ID: 0000-0003-3506-9035)

Hui Lisa (Orcid ID: 0000-0002-9720-3562)

UOG 21899

Original Article

The Victorian Perinatal Record Linkage Study

Lindquist et al.

Correspondence to: Dr L. Hui, Reproductive Epidemiology group, Murdoch Children's Research Institute,

Parkville, Victoria, Australia (e-mail: lisa.hui@unimelb.edu.au)

Accepted: 26 September 2019

\title{
State-wide utilization and performance of traditional and cell-free DNA-based prenatal testing pathways: the Victorian Perinatal Record Linkage (PeRL)
}

study
A. Lindquist ${ }^{1,2 \#}$, L. Huil ${ }^{1,2,3,4 \#}$, A. Poulton ${ }^{1}$, E. Kluckow ${ }^{1,3}$, B. Hutchinson ${ }^{2}$, M. D.
Pertile $^{5,6}$, L. Bonacquisto ${ }^{5}$, L. Gugasyan7, A. Kulkarni ${ }^{7}$, J. Harraway ${ }^{8}$, A. Howden 9 , R.

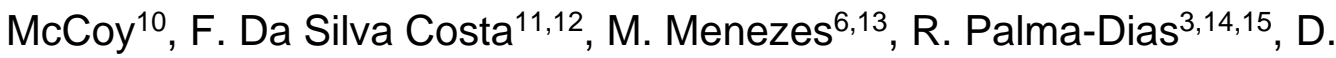
Nisbet $^{14,15,16}$, N. Martin ${ }^{17}$, M. Bethune ${ }^{3,18}$, Z. Poulakis $6,19,20$ and J. Halliday ${ }^{1,6}$

\#A.L. and L.H. contributed equally to this work.

${ }^{1}$ Reproductive Epidemiology group, Murdoch Children's Research Institute, Parkville, Victoria, Australia; ${ }^{2}$ Department of Obstetrics, Mercy Hospital for Women, Heidelberg, Victoria, Australia; ${ }^{3}$ Department of Obstetrics and Gynaecology, University of Melbourne, Parkville, Victoria, Australia; ${ }^{4}$ The Northern Hospital, Epping, Victoria, Australia; ${ }^{5}$ Victorian Clinical Genetics Services, Murdoch Children's

This is the author manuscript accepted for publication and has undergone full peer review but has not been through the copyediting, typesetting, pagination and proofreading process, which may lead to differences between this version and the Version of Record. Please cite this article as doi: 10.1002/uog.21899

This article is protected by copyright. All rights reserved. 
Research Institute, Parkville, Victoria, Australia; ${ }^{6}$ Department of Paediatrics, University of Melbourne, Parkville, Victoria, Australia; ${ }^{7}$ Cytogenetics, Monash Pathology, Monash Medical Centre, Clayton, Victoria, Australia; ${ }^{8}$ Sullivan Nicolaides Pathology, Brisbane, Queensland, Australia; ${ }^{9}$ Cytogenetics, Melbourne Pathology, Collingwood, Victoria, Australia; ${ }^{10}$ Molecular Genetics, Australian Clinical Labs, Clayton, Victoria, Australia; ${ }^{11}$ Department of Gynecology and Obstetrics, Ribeirão Preto Medical School, University of São Paulo, Ribeirão Preto, São Paulo, Brazil; ${ }^{12}$ Department of Obstetrics and Gynaecology, Monash University, Clayton, Victoria, Australia; ${ }^{13}$ Monash Ultrasound for Women, Richmond, Victoria, Australia; ${ }^{14}$ Women's Ultrasound Melbourne, East Melbourne, Victoria, Australia; ${ }^{15}$ Ultrasound Services, Royal Women's Hospital, Parkville, Victoria, Australia; ${ }^{16}$ Department of Medicine and Radiology, University of Melbourne, Parkville, Victoria, Australia; ${ }^{17}$ Virtus Diagnostics and Pathology Services, Spring Hill, Queensland, Australia; ${ }^{18}$ Specialist Women's Ultrasound, Box Hill, Victoria, Australia; ${ }^{19}$ Victorian Infant Hearing Screening Program, Centre for Community Child Health, Royal Children's Hospital, Parkville, Victoria, Australia; ${ }^{20}$ Prevention Innovation Group, Population Health, Murdoch Children's Research Institute, Parkville, Victoria, Australia

KEYWORDS: cell-free DNA; chromosome abnormality; Down syndrome screening; first trimester screening; nuchal translucency; prenatal diagnosis; prenatal screening; serum screening 


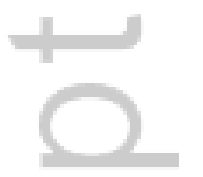




\section{+A: CONTRIBUTION}

\section{$+\mathrm{C}:$ What are the novel findings of this work?}

In a population with high uptake of prenatal screening and utilization of chromosomal microarray analysis for prenatal diagnosis, there was no statistically significant difference in the residual risk of any major chromosomal abnormality after a low-risk cell-free DNA (cfDNA) result compared with a low-risk combined first-trimester screening result.

\section{$+C$ : What are the clinical implications of this work?}

Women and clinicians can be reassured that the use of cfDNA as a first-line screening option has a low risk of missing an atypical chromosomal abnormality.

\section{$+A:$ ABSTRACT}

Objectives To perform individual record linkage of women undergoing screening with cell-free DNA (cfDNA), combined first-trimester screening (CFTS), second-trimester serum screening (STSS), and/or prenatal and postnatal cytogenetic testing with the aim to (1) obtain population-based estimates of utilization of prenatal screening and invasive diagnosis, (2) analyze the performance of different prenatal screening strategies, and (3) report the residual risk of any major chromosomal abnormality following a low-risk aneuploidy screening result. 
Methods This was a retrospective study of women residing in the state of Victoria, Australia, who underwent prenatal screening or invasive diagnosis in 2015. Patientfunded cfDNA referrals from multiple providers were merged with state-wide results for government-subsidized CFTS, STSS and invasive diagnostic procedures. Postnatal cytogenetic results from products of conception and infants up to 12 months of age were obtained to ascertain cases of false-negative screening results and atypical chromosomal abnormalities. Individual record linkage was performed using LinkageWiz $^{\mathrm{TM}}$.

Results During the study period, there were 79140 births and 66166 (83.6\%) women underwent at least one form of aneuploidy screening. Linkage data were complete for 93.5\% ( $n=61877$ ) of women who underwent screening, and of these, $73.2 \%$ ( $n=45275)$ used CFTS alone, 20.2\% $(n=12486)$ used cfDNA alone; 5.3\% $(n=3268)$ used STSS alone, $1.3 \%(n=813)$ used both CFTS and cfDNA, and $<0.1 \%$ $(n=35)$ used both STSS and cfDNA. CFTS had a combined sensitivity for trisomies 21 (T21), 18 (T18) and 13 (T13) of 89.57\% (95\% Cl, 82.64-93.93\%) for a screenpositive rate (SPR) of $2.94 \%$. There were 12 false-negative results in the CFTS pathway, comprising 10 cases of T21, one of T18 and one of T13. cfDNA had a combined sensitivity for T21, T18 and T13 of 100\% (95\% Cl, 95.00-100\%) for a SPR of $1.21 \%$. When high-risk cfDNA results for any chromosome (including the sex chromosomes) and failed cfDNA tests were treated as screen positives, the SPR for 
cfDNA increased to $2.42 \%$. The risk of any major chromosomal abnormality (including atypical abnormalities) detected on prenatal or postnatal diagnostic testing after a lowrisk screening result was 1 in 1188 for CFTS $(n=37)$ and 1 in 762 for $\operatorname{cfDNA}(n=16)$ $(P=0.13)$. The range of chromosomal abnormalities detected after a low-risk cfDNA result included pathogenic copy-number variants $(n=6)$, triploidy $(n=3)$, rare autosomal trisomies $(n=3)$ and monosomy $\times(n=2)$.

Conclusions Our state-wide record-linkage analysis delineated the utilization and clinical performance of the multitude of prenatal screening pathways available to pregnant women. The sensitivity of cfDNA for T21, T18 and T13 was clearly superior to that of CFTS. While there was no statistically significant difference in the residual risk of any major chromosomal abnormality after a low-risk CFTS or cfDNA result, there were fewer live infants diagnosed with a major chromosomal abnormality in the cfDNA cohort. These data provide valuable population-based evidence to inform practice recommendations and health policies.

\section{+A: INTRODUCTION}

The complexity of prenatal screening and diagnostic pathways has increased as a result of the introduction of cell-free DNA (cfDNA) screening, improvements in ultrasound detection of fetal abnormalities and the widespread adoption of 
chromosomal microarrays. Women and clinicians now navigate an unprecedented number of clinical pathways for the detection of fetal aneuploidy and copy-number variants (CNVs), including multiple combinations of first- and second-trimester ultrasound examinations, first- and second-trimester serum markers and cfDNA screening ${ }^{1}$.

Several countries with nationalized health systems, including the UK and Denmark, have demonstrated that cfDNA screening can be provided effectively as part of a publicly funded Down-syndrome screening program ${ }^{2-4}$. However, other countries, such as the USA, use a variety of models to deliver prenatal screening, ranging from coordinated state-funded systems to private health management organization-based programs ${ }^{5}$. Australia has a universal public healthcare system in which conventional prenatal screening and ultrasound are supported by government rebates, with the exception of cfDNA screening, which is not covered by either public or private insurers. This allows women to access conventional screening in a relatively affordable way, but with the requirement to self-fund new technologies such as cfDNA screening. To date, combined first-trimester screening (CFTS) with nuchal translucency (NT) measurement and serum markers has been the most common prenatal screening method $^{6}$. In agreement with others, we have observed a dramatic decline in invasive diagnostic tests in recent years, due to the combined effect of increasing uptake of CFTS and the introduction of $\mathrm{cfDNA}^{7}$. 
The popularity of cfDNA screening and subsequent decline in invasive diagnostic testing has stimulated debate on the missed opportunities to perform chromosomal microarray (CMA) analysis and detect pathogenic CNVs and atypical chromosomal abnormalities ${ }^{8,9}$. Existing studies comparing the detection of atypical abnormalities through traditional vs cfDNA screening pathways have been theoretical and modelled using historical prenatal diagnosis datasets ${ }^{10-12}$. In this study, we extended our prior work $^{11}$ by obtaining state-wide cfDNA and postnatal cytogenetic data to perform a record-linkage study. In doing so, we aimed to ascertain the 'real world' utilization and performance of various screening pathways, and to report the numbers of major chromosomal abnormalities detected after a low-risk CFTS or cfDNA result.

\section{+A: METHODS}

The state of Victoria in Australia has approximately 79000 births per annum, median maternal age of 31.5 years, average fertility rate of 1.7 births per woman, and an average weekly disposable household income of AUD $998^{13}$. Of women who gave birth in the state of Victoria in $2015,73.4 \%$ were public patients and $26.3 \%$ were private patients ${ }^{14}$.

The Royal Australian and New Zealand College of Obstetricians and Gynaecologists and the Human Genetics Society of Australasia recommend that all pregnant women in Australia be offered voluntary screening for fetal chromosomal and structural abnormalities ${ }^{15}$. Government rebates are available for CFTS, second-trimester serum screening (STSS) and the mid-trimester morphology ultrasound, with variable out-of- 
pocket cost to the patient (typically < AUD 200). CFTS incorporates ultrasound measurement of fetal NT (with or without assessment of the nasal bone) at $11+0$ to $13+6$ weeks' gestation, with assessment of serum pregnancy-associated plasma protein-A and free $\beta$-human chorionic gonadotropin ( $\beta$-hCG) levels at $9+0$ to $13+6$ weeks, to generate risk results for trisomies 21 (T21), 18 (T18) and 13 (T13). STSS is conducted between 14 and 20 weeks' gestation as a quadruple panel including $\alpha$-fetoprotein, unconjugated estriol, free $\beta$-hCG and dimeric inhibin A. cfDNA screening became commercially available in Australia from multiple providers in early 2013 on a patient-funded basis, at an average cost of AUD 500. The utilization of cfDNA is not subsidized by the government, but has nevertheless been rapidly adopted through individual patient choice and clinician practice, typically being performed at 10-11 weeks' gestation ${ }^{16}$. Systematic data collection on total cfDNA testing numbers in the state of Victoria has not been performed prior to this study.

Ethical approvals for this study were obtained from the human research ethics committees of the Royal Children's Hospital (35171B) and Monash Health (12063B). Our study was granted a waiver of individual patient consent, under the provisions of Section 3.2.4 of the National Health \& Medical Research Council National Statement on Ethical Conduct in Human Research.

\section{+B: Prenatal testing pathways}


Prenatal screening is discussed and offered to pregnant women by primary care physicians (general practitioners), public hospital antenatal care providers, private obstetricians or independent midwives. The prenatal testing pathways available to women in Australia in 2015 were: (1) CFTS alone; (2) cfDNA alone; (3) STSS alone; (4) CFTS and cfDNA; (5) STSS and cfDNA; (6) invasive prenatal diagnosis without prior screening test (e.g. for ultrasound abnormality, advanced maternal age, prior history or maternal request); and (7) no prenatal screening or invasive diagnostic testing.

The standard components of invasive prenatal diagnostic testing (amniocentesis and chorionic villus sampling (CVS), and associated chromosomal analysis) are fully government-funded if performed in a public hospital, and partially funded if performed in a private ultrasound practice. All women are offered a second-trimester morphology scan in addition to aneuploidy screening tests. Over $90 \%$ of pregnancies in the state of Victoria undergo a routine second-trimester morphology scan ${ }^{17}$. We have reported previously that government billing statistics for the 11-14-week ultrasound scan continued to rise after cfDNA screening became locally available in $2013^{6}$, suggesting the continued use of this scan for early anatomy survey in the cfDNA era, but individual linkage of our prenatal testing cohort with ultrasound data was not possible.

\section{+B: Inclusion criteria}


The primary inclusion criteria were women with a residential postcode within the state of Victoria who underwent a screening test or primary invasive diagnostic prenatal test (without prior screening) during the 2015 calendar year. The year 2015 was selected because it is 2 years after cfDNA became commercially available in Victoria and the last year during which serum screening was performed by a single state-wide provider.

\section{+B: Data sources}

Prenatal screening and diagnostic services are provided by multiple health services, including public hospitals, private pathology laboratories and private ultrasound practices.

CFTS and STSS serum screening results were obtained from the Victorian Clinical Genetics Service, a centralized state-wide laboratory provider. Some women may have accessed interstate laboratories for their serum testing due to their provider location (e.g. rural areas on state borders), but these are expected to comprise a small minority of cases as the Victoria state population is highly centralized in and around the capital city, Melbourne.

cfDNA results were obtained from five pathology services and three private ultrasound practices (see Acknowledgments for details). cfDNA tests included random wholegenome (percept ${ }^{\mathrm{TM}}$ ), chromosome-selective (Harmony ${ }^{\mathrm{TM}}$ ) and single-nucleotide polymorphism-based (Panorama ${ }^{T M}$ ) assays. While we could not ensure $100 \%$ 
ascertainment due to the fragmented nature of cfDNA provision in Australia, the participating laboratories and ultrasound services collectively captured the vast majority of cfDNA tests performed in the state of Victoria.

State-wide prenatal invasive diagnostic testing data (amniocentesis and CVS) were obtained from the four cytogenetic laboratories that contribute annually to the Victorian Prenatal Diagnosis Database. This database has been described in detail elsewhere ${ }^{18}$.

Data on postnatal diagnostic testing (chromosomal analysis of products of conception (POC), stillbirths and live infants aged 12 months or less) were obtained from the three cytogenetic laboratories in the state of Victoria that offered this service during the study period. Chromosomal analysis of a miscarriage is not performed routinely, but is offered for recurrent miscarriage (three or more unexplained first-trimester losses), for a miscarriage after 14 weeks' gestation, and for all stillbirths (defined as birth of a baby without signs of life after 20 weeks' gestation or with a birth weight $\geq 400 \mathrm{~g}$ if the gestational age is unknown). The vast majority of chromosomal analyses are performed using CMA analysis.

Total births for the study cohort were estimated using the Consultative Council on Obstetric and Paediatric Morbidity and Mortality data for 2015 and 2016, which includes all registered births $\geq 20$ weeks' gestation ${ }^{14,19}$. As women accessing prenatal 
screening in 2015 would be expected to give birth from mid-2015 to mid-2016, the average of annual births for 2015 and 2016 was used to estimate the number of births for the 2015 screening cohort.

Data collection is summarized in Figure 1. To avoid including women who underwent prenatal screening at the end of 2014 , records of women who had follow-up prenatal testing in January 2015 due to a high-risk screening result in 2014 were excluded from the dataset. Similarly, the 'tail' end of the dataset in January and February 2016 was reviewed manually to include women who had a screening test in 2015 , and were then followed up with further tests in early 2016.

\section{+B: Data coding}

$+\mathrm{C}:$ Screening tests

The following data were obtained for each prenatal case: maternal age, indication(s) for screening and/or diagnostic testing, type of test performed, gestational age at time of testing, test date, test result, and whether the pregnancy was singleton or multiple.

CFTS results were coded as (1) high risk (risk $\geq 1$ in 300 for T21, $\geq 1$ in 175 for T18 and $\geq 1$ in 100 for T13), (2) low risk, or (3) unknown risk or missing data. cfDNA results were coded as high risk, low risk, failed results ('no call') or canceled (not performed). STSS results were coded as high risk if the risk of T21 was $\geq 1$ in 250 or the risk of T18 was $\geq 1$ in 200 . Women with unknown risk/missing data were those who had 
undergone serum screening but had incorrect dates, or who did not have a NT measurement supplied to the serum screening laboratory for completion of CFTS risk calculation.

\section{$+\mathrm{C}$ : Diagnostic tests}

The types of chromosomal analysis included were G-banded karyotyping and CMA. The CMA analyses in use by the various laboratories during the study period were high-resolution whole genome single-nucleotide polymorphism (SNP) microarrays (Affymetrix Cytoscan 750K array (Thermo Fisher Scientific, Waltham, MA, USA), Illumina CytoSNP-12 array (Illumina, Inc., San Diego, CA, USA), and the Agilent SurePrint Custom G3 CGH+SNP (60K) (Agilent Technologies, Santa Clara, CA, USA)).

Results of chromosomal analyses were categorized as normal or abnormal. Abnormal results were further classified into 'major' and 'minor' abnormalities. Major chromosomal abnormalities included all cases of autosomal and sex chromosome aneuploidy, polyploidy, unbalanced translocations/rearrangements, level-III mosaics, pathogenic CNVs and likely pathogenic CNVs. CNVs were classified according to the guidelines of the American College of Medical Genetics (ACMG) ${ }^{20}$. CNVs were classified as 'pathogenic' when they encompassed a region implicated in a welldescribed abnormal phenotype, as documented in multiple peer-reviewed publications. 'Likely pathogenic' variants were CNVs that met the ACMG definitions of a CNV 'described in a single case report but with well-defined breakpoints and 
phenotype, both specific and relevant to the patient findings', or CNV interval 'with a very compelling gene function that is relevant and specific to the reason for patient referral'.

Minor chromosomal abnormalities included confined placental mosaicism, balanced translocations/rearrangements, and CNVs of uncertain or unknown significance (including susceptibility loci and low penetrance CNVs). These minor abnormalities are not reported in this study.

\section{+B: Data linkage}

Each dataset was individually cleaned and re-coded using uniform nomenclature before merging was performed. The datasets were probabilistically linked in a stepwise manner using LinkageWiz ${ }^{T M}$ (LinkageWiz Software, Payneham, South Australia, Australia; http://www.linkagewiz.net/). LinkageWiz is a propriety software tool used for probabilistic data matching between large datasets. The date of birth, name and postcode of the mother were used as identifiers to link the prenatal screening, invasive prenatal diagnosis and postnatal diagnosis datasets of the patient. If required, additional identifiers, such as maternal age and date of testing, and manual clerical review were used to classify potential matches. Linkage 1 was performed between STSS or CFTS serum screening and cfDNA data, and Linkage 2 was performed between Linkage 1 and prenatal invasive diagnostic testing data (Figure 1). For Linkage 3, abnormal postnatal infant results were linked to maternal identifiers in the 
prenatal datasets through the assistance of the Victorian Infant Hearing Screening program $^{21}$. At each step, potential data matches were manually examined and confirmed or rejected using the clerical review tool in LinkageWiz.

\section{$+B$ : Statistical analysis}

The performance of CFTS, cfDNA and STSS for the detection of T21 and of T21T13-T18 combined was assessed using sensitivity, specificity and screen-positive rate (SPR). Sensitivity was defined as the probability of a high-risk screening result in an affected pregnancy [true positives/(true positives + false negatives)]. Specificity was the probability of a low-risk screening result in an unaffected pregnancy [true negatives/(true negatives + false positives)]. SPR was defined as the percentage of screening results that returned a high-risk result (true positive and false positive results). For women who underwent cfDNA screening, the SPR was calculated both with and without inclusion of the failed cfDNA calls. A SPR including failed cfDNA results was calculated because, in practice, failed cfDNA results can generate additional consultations, genetic counselling and further fetal assessment in a similar manner to a high-risk result 22 .

The prenatal diagnosis rate was defined as the number of prenatal invasive diagnostic procedures undertaken following a screening test (regardless of whether the indication for invasive testing was a high-risk screening result or other, such as structural abnormality). The diagnostic yield was defined as the number of major abnormalities detected as a percentage of the total invasive diagnostic tests performed. False- 
negative screening results were defined as cases within our linked dataset with a confirmed diagnosis of T21, T18 or T13 on prenatal or postnatal diagnostic testing, following a prior low-risk screening result. Manual notifications of false-negative screening tests to individual laboratories by clinicians were also included.

Descriptive analysis was performed using STATA version 14 (StataCorp LP, College Station, TX, USA). Differences in proportions were assessed using the chi-square test with a statistically significant $P$-value threshold of 0.05 .

\section{+A: RESULTS}

In 2015, there were 79140 births in the state of Victoria and 66166 women had at least one prenatal screening test, representing an overall screening uptake rate of 83.6\%. Screening data were complete for $93.5 \%(61877 / 66,166)$ of the women who underwent any form of prenatal screening, and these were assigned as the 'matched cohort' (Figure 2). Of the 61877 women in the matched screening cohort, $73.2 \%$ ( $n=45275)$ used CFTS alone, 20.2\% $(n=12,486)$ used cfDNA alone; 5.3\% ( $n=3268)$ used STSS alone, 1.3\% $(n=813)$ used both CFTS and cfDNA, and $<0.1 \%$ $(n=35)$ used both STSS and cfDNA (Figure 2).

In total, 1975 invasive prenatal diagnostic tests were included (matched screening cohort $n=1197$, primary prenatal diagnosis $n=693$, unmatched cohort $n=85$ ), of 
which $83.8 \%$ were performed using CMA analysis. The total number of major chromosomal abnormalities detected via invasive prenatal diagnosis was $378(0.48 \%$ of births).

\section{+B: Excluded and unmatched data}

Screening results were incomplete for 4289 women. This was most commonly due to the NT measurement not being provided to the serum laboratory for calculation of

CFTS risk, or gestational age being outside the window for CFTS $(11+0$ to $13+6$ weeks). These women were excluded from subsequent analyses (Figure 2).

There were 40 samples that had matched abnormal prenatal and postnatal diagnosis results, representing duplicate testing for the same pregnancy. These results were analyzed as part of the prenatal diagnosis group and their counts removed from the postnatal diagnosis group.

In $0.1 \%(n=85)$ of the total cohort, prenatal diagnosis was performed for a high risk screening result that we could not match in our prenatal screening dataset (Figure 2). The results of diagnostic testing for this 'unmatched cohort' are reported separately in Table S1.

\section{+B: Utilization of prenatal testing pathways}

The results of prenatal and postnatal diagnostic testing in the matched cohort are presented in Table 1. 
+C: Prenatal testing pathway 1: CFTS alone

CFTS alone was the most commonly used screening strategy, utilized by 45275 women. The median maternal age was 31 years and median gestational age at the time of serum collection was 11 (range 9-14) weeks, with NT measurement being performed later at $11+0$ to $13+6$ weeks' gestation. Of these women, $2.9 \%$ ( $n=1329)$ had a high-risk result $(\geq 1$ in 300). In this high-risk group, the invasive prenatal diagnosis rate was $42.3 \%$ (562/1329) and the diagnostic yield was $23.1 \%$ (130/562) (Table 1). In a further 41 women with a high-risk CFTS result who did not have prenatal diagnostic testing, postnatal testing confirmed a major chromosomal abnormality in 56.0\% (23/41).

The overall sensitivity of CFTS for T21, T13 and T18 was 89.6\% (103/115) for a SPR of $2.94 \%$ (Table 2).

Of the 43946 women with a CFTS risk lower than 1 in 300, 0.95\% $(n=417)$ had subsequent prenatal $(n=265)$ or postnatal $(n=152)$ diagnostic testing, of which $8.9 \%$ ( $n=37$ ) had an abnormal result (Table 3). These included 12 false-negative results, comprising 10 cases of T21, one of T18 and one of T13. Further details on these falsenegative cases are provided in Tables S2 and S3. The most common type of abnormality detected after a low-risk CFTS result was a pathogenic CNV $(n=14)$, including eight 22q11.2 microdeletions. 
$+\mathrm{C}$ : Prenatal testing pathway 2: cfDNA alone

Primary cfDNA screening was the second most common screening pathway, utilized by 12486 women. The median maternal age was 34 years and median gestational age at testing was 10 (range 9-32) weeks.

There were 235 women who received a high-risk result and 67 who received a failed cfDNA result. Of the women with a high-risk result, 48.5\% $(n=114)$ underwent invasive prenatal diagnostic testing and $12.8 \%(n=30)$ had postnatal testing, giving an overall diagnostic confirmation rate of $61.3 \%(144 / 235)$ (Table 1$)$. Of the 67 women with a failed cfDNA result, 16 proceeded to prenatal diagnosis and four of these had an abnormal result (triploidy $(n=2)$, mosaicism $(n=2))$.

The rate of invasive prenatal diagnosis following a high-risk cfDNA result varied according to the suspected aneuploidy, and was highest for suspected T21 (58.7\%, 61/104). Compared with the group with a high-risk T21 result, the prenatal diagnosis rate for a high-risk T18 or T13 cfDNA result was not significantly different $(46.8 \%$ (22/47); $P=0.2)$, but it was significantly lower for suspected SCA at $36.4 \%(28 / 77)$ (Fisher's, $P<0.001)$.

The overall sensitivity of cfDNA for the common aneuploidies (T21, T13 and T18) was $100.0 \%(73 / 73)$ for a SPR of $1.21 \%$ (Table 2$)$. Nine high-risk cfDNA results for T21/T13/T18 were verified as false positives on postnatal diagnostic testing, 
comprising two high-risk results for T21 and seven for T18 or T13. When high-risk results for additional chromosomal abnormalities and failed cfDNA results were counted as screen positives, the SPR was $2.42 \%$. The overall diagnostic yield of invasive prenatal diagnostic testing for a high-risk cfDNA result was $60.0 \%(78 / 130)$.

$+C$ : Prenatal testing pathway 3: STSS alone

STSS alone was utilized by 3268 women. The median maternal age was 29 years and median gestational age was 15 (14-20) weeks. One case of T21 and two of T18 were detected by STSS and confirmed by invasive prenatal diagnosis. There was one falsenegative STSS result for T18 that was subsequently detected via prenatal diagnosis performed for ultrasound abnormality. One additional false-negative case of T21 was detected postnatally. The sensitivity of STSS for T21 and T18 was 60\% (3/5), and the diagnostic yield of invasive prenatal diagnosis in this group was 7.0\% (7/100).

$+C$ : Comparative performance of prenatal testing pathways 1, 2 and 3

Table 2 summarizes the sensitivity, specificity and SPR for T21, T18 and T13 for CFTS alone, cfDNA alone and STSS alone. The detection rate for the common trisomies was highest in the cfDNA cohort (100.0\%) compared with the CFTS (89.6\%) and STSS $(60.0 \%)$ cohorts $(P \leq 0.001)$.

Considering the prenatal and postnatal diagnoses of all major chromosomal abnormalities, the risk of a major chromosomal abnormality being detected after a low- 
risk CFTS result $(0.084 \%, 1$ in 1188) was not significantly different from that after a low-risk cfDNA screening result $(0.13 \%, 1$ in 762$)\left(x^{2}=2.246, P=0.13\right.$; Table 3$)$. The most common indication for prenatal diagnosis after a low-risk CFTS or cfDNA result was fetal ultrasound abnormalities. Postnatal testing was most commonly performed on products of conception. None of the three triploid pregnancies that had a low-risk cfDNA result utilized a SNP-based cfDNA assay that would have been expected to detect triploidy. Details of the cases with abnormal chromosomal findings detected following a low-risk CFTS or cfDNA result are presented in Tables S4 and S5, respectively.

$+\mathrm{C}$ : Prenatal testing pathway 4: CFTS and cfDNA In our matched cohort, 813 women underwent both CFTS and cfDNA screening. Median maternal age was 34 years, median gestational age at serum screening was 10 (range 9-14) weeks and median gestational age at cfDNA was 12 (range 936) weeks. Of the 178 women with a high-risk CFTS result, 13 also received a highrisk cfDNA result. In total, 24 of the women with a high-risk CFTS result proceeded to have invasive prenatal diagnosis and $10(41.7 \%)$ of these had a major chromosomal abnormality confirmed (Table 1).

Of the 145 women who had cfDNA in the presence of a CFTS risk between 1 in 301 and 1 in 1000 ('intermediate risk'), three had a high risk cfDNA result. One case of 
trisomy 21 and one atypical anomaly were confirmed on prenatal diagnosis following a high-risk cfDNA result in this intermediate-risk group.

+C: Prenatal testing pathway 5: STSS and cfDNA

Thirty-five women had cfDNA testing after STSS, 25 after a high-risk STSS result and 10 after a low-risk STSS result. Of the women with a low-risk STSS result, eight had a low-risk cfDNA result and one had a failed result. Of the women with a high-risk STSS result, 24 had a low-risk cfDNA result and one had a failed result. One woman with a high-risk STSS result and failed cfDNA proceeded to invasive prenatal diagnosis, and received a normal diagnostic result.

$+\mathrm{C}$ : Prenatal testing pathway 6: primary invasive prenatal diagnosis without prior screening

During the study period, 693 women underwent primary invasive prenatal diagnosis, most commonly for first trimester ultrasound abnormality $(n=278)$, risk of single-gene disorder $(n=121)$ and advanced maternal age $(n=74)$. In total, 87 major chromosomal abnormalities were detected during pregnancy via this pathway, comprising $23.0 \%$ of the 378 total prenatal chromosomal abnormalities in our study cohort. The common trisomies (T21, T18 and T13) made up less than half of the abnormalities ascertained in this group (Table S6).

$+\mathrm{C}$ : Prenatal testing pathway 7: no prenatal screening or invasive diagnostic testing 
All live infants up to 12 months of age with a postnatal diagnosis of a major chromosomal abnormality were cross-checked with our merged prenatal screening and diagnosis dataset for evidence of prior prenatal screening or invasive diagnosis. We identified 24 infants with trisomy 21 that had no record of prenatal screening or diagnosis in our dataset (1 in 3300 births). Other infants in this group included 11 with 22q11.2 deletion syndrome, 11 with SCA and 37 with other pathogenic CNVs.

\section{$+\mathrm{A}$ : DISCUSSION}

Our unique perinatal record linkage collaboration of public and private stakeholders enabled us to assess multiple prenatal screening strategies in a real-world setting. Our findings confirm that CFTS performs as expected with a sensitivity of $89.6 \%$ for the detection of common aneuploidies. The SPR for CFTS was lower than previously reported $(2.9 \% \text { in our study compared with } 4.5 \% \text { in 2003-2004) })^{23}$, which we hypothesize to be attributable to the introduction of the nasal bone marker in 2011 . cfDNA had the best detection for T21, T13 and T18 (100\%), but its overall SPR (including failed cfDNA results) was only $0.5 \%$ lower than that of CFTS $(2.4 \%$ vs 2.9\%). In practice, failed cfDNA results generate additional medical costs and raise patient anxiety in a similar way to high-risk screening results, due to their association with an increased risk of aneuploidy ${ }^{24,25}$. This is an important consideration when comparing cfDNA and CFTS as primary screening tests, as it demonstrates that the high specificity of cfDNA is not fully realized in clinical practice. 
While cfDNA had $100 \%$ sensitivity for the detection of the common autosomal trisomies, the risk of a subsequent diagnosis of any major chromosomal abnormality after a low-risk cfDNA result was not statistically different from that after a low-risk CFTS result. This finding may alleviate concerns regarding the loss of ascertainment of atypical abnormalities due to increasing uptake of cfDNA $^{9}$. To our knowledge, our study is the first population-based report to reveal the actual, rather than modelled, set of selected abnormalities missed by CFTS and cfDNA screening up to 12 months after birth (notwithstanding the fact that some of those anomalies may not have a clinically detectable phenotype by that age). Importantly, though the numerical risk was not statistically significant, there were qualitative differences in the types of abnormalities missed by the two screening pathways. The majority of the abnormalities missed by cfDNA were detected via invasive prenatal diagnosis before 21 weeks' gestation, and/or were associated with pregnancy loss (i.e. triploidy, trisomies 14, 15 and 6). The only major chromosomal abnormalities detected in live infants after a low-risk cfDNA result were two pathogenic CNVs. In contrast, the abnormalities missed by CFTS included 10 cases of trisomy 21 , of which five were in live infants. Therefore, if the primary purpose of prenatal screening is to detect trisomy 21 , cfDNA is clearly the superior screening test when compared with CFTS. With the increasing availability of genome-wide cfDNA screening ${ }^{26}$, the rate of undiagnosed atypical abnormalities after cfDNA is expected to decrease, though with a likely trade-off of a higher SPR. 
Fewer than $1 \%$ of all women proceeded directly to invasive prenatal diagnosis without accessing prior screening. However, this pathway contributed almost one-quarter of the total prenatal chromosomal abnormalities, mainly due to the high diagnostic yield of prenatal diagnosis for ultrasound abnormalities ${ }^{27}$. These results highlight the important role of ultrasound for detecting chromosomal abnormalities, including atypical abnormalities, and supports current recommendations for offering diagnostic testing with CMA analysis for fetal abnormalities regardless of a prior screening 28,29 .

An unexpected finding of our study was the relatively low rate of invasive prenatal diagnosis following a high-risk cfDNA result $(114 / 235,48.5 \%)$. There are several potential explanations for this finding. Some high-risk cfDNA results might be accompanied by fetal abnormalities that may be managed based on ultrasound findings alone. The prenatal diagnosis rate also varied according to the suspected chromosomal abnormality, with pregnancies with a high-risk result for SCA having a significantly lower rate of diagnostic testing than those with suspected T21. We were unable to determine if any women proceeded directly to termination of pregnancy without ruling out the possibility of a false positive cfDNA result. Australian guidelines strongly recommend diagnostic testing prior to any management decisions, but our study was unable to assess compliance with this recommendation ${ }^{15}$.

Our ability to draw conclusions about the clinical impact of prenatal screening was limited by the lack of linkage to government data on perinatal outcomes. We therefore 
could not estimate the rate of termination of pregnancy or perinatal loss, and could only ascertain the estimated number of births with selected genomic imbalances using live infant cases in our postnatal diagnostic dataset. Miscarriages are not universally tested by chromosomal analysis in the state of Victoria, though this it is commonly performed in cases of recurrent miscarriage and is recommended for the investigation of all stillbirths ${ }^{30}$. Our residual risk calculation for missed abnormalities is therefore compromised by the $<100 \%$ ascertainment of pregnancy losses. Furthermore, the unexpectedly low prenatal diagnosis rate among patients with 'positive' screening results, and the delayed clinical presentation (beyond the first year after birth) of some relevant conditions, such as $22 q 11.2 \mathrm{DS}$, also impede our ability to calculate the real absolute residual risk of each of the screening pathways.

Our data were also limited by the incomplete ascertainment of all screening test results. The number of prenatal diagnostic tests in the unmatched screening group ( $n=85$ ) suggests that we may be missing approximately $7 \%$ of screening data due to interstate boundaries or non-participation of cfDNA referrers, which we considered acceptable given the logistical challenges of performing population-based studies in a rapidly changing environment.

As this was an observational study, selection biases and differences in background aneuploidy risk are assumed to exist in each pathway, as evident in the differences in maternal and gestational age between the primary cfDNA, CFTS and STSS groups. We also lacked the ability to determine the prior utilization of ultrasound by women in each pathway, due to the absence of state-wide data on prenatal ultrasound. We 
therefore did not report positive predictive values for each prenatal testing pathway as these are influenced by the background prevalence of the condition. The generalizability of our results to other populations should therefore be interpreted with caution.

In conclusion, our state-wide linkage analysis made it possible to compare the clinical performance of the multitude of prenatal screening pathways currently available to pregnant women. The sensitivity of cfDNA for trisomies 21,18 and 13 was clearly superior to that of CFTS. While there was no statistically significant difference in the residual risk of any major chromosomal abnormality after low-risk CFTS or cfDNA result, there were fewer live infants diagnosed with a major chromosomal abnormality in the cfDNA cohort.

\section{+A: ACKNOWLEDGMENTS}

Members of the Victorian Perinatal Record Linkage (PeRL) Collaboration. We wish to acknowledge the support of following organizations for their participation in the PeRL study. The Victorian Clinical Genetics Services (VCGS), Monash Pathology, Australian Clinical Laboratories, and Melbourne Pathology contributed the prenatal and postnatal diagnosis data. The serum screening laboratory at VCGS provided the CFTS and STSS data. cfDNA data were provided by Melbourne Pathology, Monash Pathology, Monash Ultrasound for Women, Women's Ultrasound Melbourne, 
Melbourne IVF (Virtus Diagnostics), Specialist Women's Ultrasound and VCGS. We thank the Victorian Infant Hearing Screening Program for assisting with matching of infant and maternal identifiers for prenatal and postnatal data linkage.

\section{$+B$ : Funding}

L.H. is funded by a National Health and Medical Research Council Early Career Fellowship (1105603) and J.H. was funded by a National Health and Medical Research Council Senior Research Fellowship (10121252). The funding body had no role in the conduct of the research or the writing of the manuscript.

\section{+B: Disclosures}

R.P.-D. reports a commercial relationship with Roche Diagnostics and Natera, personal fees from Philips Ultrasound, outside the submitted work. D.N. reports a commercial relationship with Roche Diagnostics and Natera, outside the submitted work.

\section{+A: REFERENCES}

1. Hui L, Bianchi DW. Noninvasive prenatal DNA testing: The vanguard of genomic medicine. Annu Rev Med 2017; 68: 459-472.

2. Oepkes D, Page-Christiaens GC, Bax CJ, Bekker MN, Bilardo CM, Boon EM, Schuring-Blom GH, Coumans AB, Faas BH, Galjaard RH, Go AT, Henneman L, Macville MV, Pajkrt E, Suijkerbuijk RF, Huijsdens-van Amsterdam K, Van Opstal D, Verweij EJ, Weiss MM, Sistermans EA; Dutch NIPT Consortium. Trial by Dutch 
laboratories for evaluation of non-invasive prenatal testing. Part I—clinical impact. Prenat Diagn 2016; 36: 1083-1090.

3. Miltoft CB, Rode L, Ekelund CK, Sundberg K, Kjaergaard S, Zingenberg H, Tabor A. Contingent first-trimester screening for aneuploidies with cell-free DNA in a Danish clinical setting. Ultrasound Obstet Gynecol 2018; 51: 470-479.

4. Chitty LS, Wright D, Hill M, Verhoef TI, Daley R, Lewis C, Mason S, McKay F, Jenkins L, Howarth A, Cameron L, McEwan A, Fisher J, Kroese M, Morris S. Uptake, outcomes, and costs of implementing non-invasive prenatal testing for Down's syndrome into NHS maternity care: prospective cohort study in eight diverse maternity units. BMJ 2016; 354: i3426.

$<O T H E R>5$. Anthem Blue Cross Blue Shield. Cell-free fetal DNA-based prenatal testing. Document no. GENE.00026, 2018.

6. Hui L, Hutchinson B, Poulton A, Halliday J. Population-based impact of noninvasive prenatal screening on screening and diagnostic testing for fetal aneuploidy. Genet Med 2017; 19: 1338-1345.

7. Robson SJ, Hui L. National decline in invasive prenatal diagnostic procedures in association with uptake of combined first trimester and cell-free DNA aneuploidy screening. Aust N Z J Obstet Gynaecol 2015; 55: 507-510.

8. Vogel I, Petersen OB. Prenatal screening for atypical chromosomal abnormalities: past or future? Ultrasound Obstet Gynecol 2018; 51: 434-435. 
9. Evans MI, Andriole S, Curtis J, Evans SM, Kessler AA, Rubenstein AF. The epidemic of abnormal copy number variant cases missed because of reliance upon noninvasive prenatal screening. Prenat Diagn 2018; 38: 730-734.

10. Evans MI, Evans SM, Bennett TA, Wapner RJ. The price of abandoning diagnostic testing for cell-free fetal DNA screening. Prenat Diagn 2018; 38: 243-245.

11. Lindquist A, Poulton A, Halliday J, Hui L. Prenatal diagnostic testing and atypical chromosome abnormalities following combined first-trimester screening: implications for contingent models of non-invasive prenatal testing. Ultrasound Obstet Gynecol 2018; 51: 487-492.

12. Petersen OB, Vogel I, Ekelund C, Hyett J, Tabor A; Danish Fetal Medicine Study Group; Danish Clinical Genetics Study Group. Potential diagnostic consequences of applying non-invasive prenatal testing: population-based study from a country with existing first-trimester screening. Ultrasound Obstet Gynecol 2014; 43: 265271.

$<$ EPATH $>13$. Australian Bureau of Statistics. ABS.Stat ${ }^{\text {beta }}$. 2019. http://stat.data.abs.gov.au/

$<$ OTHER $>14$. The Consultative Council on Obstetric and Paediatric Mortality and Morbidity. Victoria's mothers, babies and children 2014 and 2015. Melbourne, Victoria: Department of Health and Human Services, 2017.

$<$ OTHER $>15$. The Royal Australian and New Zealand College of Obstetricians and Gynaecologists and Human Genetics Society of Australasia. Prenatal Screening and diagnostic testing for fetal chromosomal and genetic conditions. C-Obs 59. 
The Royal Australian and New Zealand College of Obstetricians and Gynaecologists: Melbourne, Victoria, Australia, 2018.

16. McLennan A, Palma-Dias R, da Silva Costa F, Meagher S, Nisbet DL, Scott F. Noninvasive prenatal testing in routine clinical practice - an audit of NIPT and combined first-trimester screening in an unselected Australian population. Aust $N$ Z J Obstet Gynaecol 2016; 56: 22-28.

$<$ OTHER $>17$. Medicare Australia. Medicare Item Reports. Item number 44706. Australian Government: Canberra, Australian Capital Territory, 2019.

18. Hui L, Muggli EE, Halliday JL. Population-based trends in prenatal screening and diagnosis for aneuploidy: a retrospective analysis of 38 years of state-wide data. BJOG 2016; 123: 90-97.

$<$ OTHER $>$ 19. The Consultative Council on Obstetric and Paediatric Mortality and Morbidity. Victoria's mothers, babies and children 2016. Department of Health and Human Services: Melbourne, Victoria, Australia, 2017.

20. Kearney HM, Thorland EC, Brown KK, Quintero-Rivera F, South ST; Working Group of the American College of Medical Genetics Laboratory Quality Assurance Committee. American College of Medical Genetics standards and guidelines for interpretation and reporting of postnatal constitutional copy number variants. Genet Med 2011; 13: 680-685.

$<$ EPATH $>21$. The Royal Children's Hospital. About the Victorian Infant Hearing Screening Program VIHSP. https: //www.rch.org.au/vihsp/. 
22. Gregg AR, Skotko BG, Benkendorf JL, Monaghan KG, Bajaj K, Best RG, Klugman S, Watson MS. Noninvasive prenatal screening for fetal aneuploidy, 2016 update: a position statement of the American College of Medical Genetics and Genomics. Genet Med 2016; 18: 1056-1065.

23. Jaques AM, Collins VR, Muggli EE, Amor DJ, Francis I, Sheffield LJ, Halliday JL. Uptake of prenatal diagnostic testing and the effectiveness of prenatal screening for Down syndrome. Prenat Diagn 2010; 30: 522-530.

24. Norton ME, Baer RJ, Wapner RJ, Kuppermann M, Jelliffe-Pawlowski LL, Currier RJ. Cell-free DNA vs sequential screening for the detection of fetal chromosomal abnormalities. Am J Obstet Gynecol 2016; 214: 727.e1-6.

25. Palomaki GE, Kloza EM, Lambert-Messerlian GM, van den Boom D, Ehrich M, Deciu C, Bombard AT, Haddow JE. Circulating cell free DNA testing: are some test failures informative? Prenat Diagn 2015; 35: 289-293.

26. Pertile MD, Halks-Miller M, Flowers N, Barbacioru C, Kinnings SL, Vavrek D, Seltzer WK, Bianchi DW. Rare autosomal trisomies, revealed by maternal plasma DNA sequencing, suggest increased risk of feto-placental disease. Sci Trans/ Med 2017; 9: eaan1240.

27. Lostchuck E, Poulton A, Halliday J, Hui L. Population-based trends in ultrasoundindicated prenatal diagnosis from 1994 to 2016: two decades of change. Ultrasound Obstet Gynecol 2019; 53: 503-511.

28. Salomon LJ, Alfirevic Z, Audibert F, Kagan KO, Paladini D, Yeo G, Raine-Fenning N; ISUOG Clinical Standards committee. ISUOG updated consensus statement 
on the impact of cfDNA aneuploidy testing on screening policies and prenatal ultrasound practice. Ultrasound Obstet Gynecol 2017; 49: 815-816.

29. Wapner RJ, Martin CL, Levy B, Ballif BC, Eng CM, Zachary JM, Savage M, Platt LD, Saltzman D, Grobman WA, Klugman S, Scholl T, Simpson JL, McCall K, Aggarwal VS, Bunke B, Nahum O, Patel A, Lamb AN, Thom EA, Beaudet AL, Ledbetter DH, Shaffer LG, Jackson L. Chromosomal microarray versus karyotyping for prenatal diagnosis. N Engl J Med 2012; 367: 2175-2184.

$<$ EPATH $>30$. Perinatal Society of Australia and New Zealand. Clinical practice guideline for care around stillbirth and neonatal death, 2018. <https://sand.psanz.com.au/clinical-practice/clinical-guidelines>.

\section{+A: SUPPORTING INFORMATION ON THE INTERNET}

The following supporting information may be found in the online version of this article:

Table S1 Results of prenatal diagnosis in unmatched high-risk screening cohort Table S2 Prenatally diagnosed false-negative screening results for trisomies 21, 18 and 13 in patients who underwent CFTS alone

Table S3 Postnatally diagnosed false-negative screening results for trisomies 21, 18 and 13 in patients who underwent CFTS alone

Table S4 Chromosomal abnormalities diagnosed prenatally or postnatally following a low-risk result on CFTS

Table S5 Chromosomal abnormalities diagnosed prenatally or postnatally following a low-risk cfDNA result

Table S6 Major chromosomal abnormalities detected following primary diagnostic testing 


\section{Figure legends}

Figure 1 Overview of individual record linkage process used in study. cfDNA, cellfree DNA; CFTS, combined first-trimester screening; CVS, chorionic villus sampling; STSS, second-trimester serum screening.

Figure 2 Utilization of prenatal testing pathways in state of Victoria in 2015 and detection through each pathway of major chromosomal abnormalities confirmed by invasive prenatal diagnosis (PND). Matched cohort included in record linkage analysis is indicated by gray shading. *Ultrasound abnormality (US abn): $n=278$ in first trimester; $n=77$ in second or third trimester. †Indications for PND in unmatched cohort were: CFTS in 35 women, STSS in seven, cfDNA in 40 and unspecified highrisk screening result in three. ‡Major chromosomal abnormality in live infants only. cfDNA, cell-free DNA; CFTS, combined first-trimester screening; STSS, secondtrimester serum screening. 


\section{University Library}

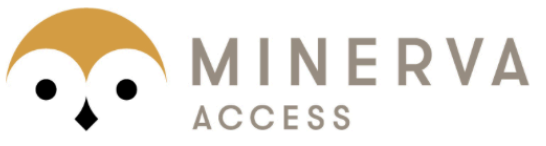

\section{A gateway to Melbourne's research publications}

Minerva Access is the Institutional Repository of The University of Melbourne

\section{Author/s:}

Lindquist, A; Hui, L; Poulton, A; Kluckow, E; Hutchinson, B; Pertile, MD; Bonacquisto, L;

Gugasyan, L; Kulkarni, A; Harraway, J; Howden, A; Mccoy, R; Da Silva Costa, F; Menezes, M; Palma-Dias, R; Nisbet, D; Martin, N; Bethune, M; Poulakis, Z; Halliday, J

Title:

State-wide utilization and performance of traditional and cell-free DNA-based prenatal testing pathways: the Victorian Perinatal Record Linkage (PeRL) study

Date:

2020-08-01

\section{Citation:}

Lindquist, A., Hui, L., Poulton, A., Kluckow, E., Hutchinson, B., Pertile, M. D., Bonacquisto, L., Gugasyan, L., Kulkarni, A., Harraway, J., Howden, A., Mccoy, R., Da Silva Costa, F., Menezes, M., Palma-Dias, R., Nisbet, D., Martin, N., Bethune, M., Poulakis, Z. \& Halliday, J. (2020). State-wide utilization and performance of traditional and cell-free DNA-based prenatal testing pathways: the Victorian Perinatal Record Linkage (PeRL) study.

ULTRASOUND IN OBSTETRICS \& GYNECOLOGY, 56 (2), pp.215-224. https://doi.org/10.1002/uog.21899.

\section{Persistent Link:}

http://hdl.handle.net/11343/276093

\section{File Description:}

Accepted version 